\title{
Proximal normal structure and relatively nonexpansive mappings
}

\author{
by
A. Anthony Eldred (Chennai), W. A. Kirk (Iowa City, IA) and P. Veeramani (Chennai)

\begin{abstract}
The notion of proximal normal structure is introduced and used to study mappings that are "relatively nonexpansive" in the sense that they are defined on the union of two subsets $A$ and $B$ of a Banach space $X$ and satisfy $\|T x-T y\| \leq\|x-y\|$ for all $x \in A, y \in B$. It is shown that if $A$ and $B$ are weakly compact and convex, and if the pair $(A, B)$ has proximal normal structure, then a relatively nonexpansive mapping $T: A \cup B \rightarrow A \cup B$ satisfying (i) $T(A) \subseteq B$ and $T(B) \subseteq A$, has a proximal point in the sense that there exists $x_{0} \in A \cup B$ such that $\left\|x_{0}-T x_{0}\right\|=\operatorname{dist}(A, B)$. If in addition the norm of $X$ is strictly convex, and if (i) is replaced with (i) $T(A) \subseteq A$ and $T(B) \subseteq B$, then the conclusion is that there exist $x_{0} \in A$ and $y_{0} \in B$ such that $x_{0}$ and $y_{0}$ are fixed points of $T$ and $\left\|x_{0}-y_{0}\right\|=\operatorname{dist}(A, B)$. Because every bounded closed convex pair in a uniformly convex Banach space has proximal normal structure, these results hold in all uniformly convex spaces. A Krasnosel'skiu type iteration method for approximating the fixed points of relatively nonexpansive mappings is also given, and some related Hilbert space results are discussed.
\end{abstract}

1. Introduction. Let $X$ be a normed linear space and $D \subseteq X$. Recall that a mapping $T: D \rightarrow D$ is nonexpansive if $\|T x-T y\| \leq\|x-y\|$ for all $x, y \in D$. In this paper we consider mappings that are "relatively nonexpansive" in the sense that they are defined on the union of two subsets $A$ and $B$ of $X$ and satisfy $\|T x-T y\| \leq\|x-y\|$ for all $x \in A, y \in B$. We introduce the notion of "proximal normal structure", and we show that if $A$ and $B$ are weakly compact and convex, and the pair $(A, B)$ has proximal normal structure, then every relatively nonexpansive mapping $T: A \cup B \rightarrow A \cup B$ for which $T(A) \subseteq B$ and $T(B) \subseteq A$ has a best proximity point. This means that there exists $x \in A \cup B$ such that $\|x-T x\|=\operatorname{dist}(A, B)$. As a companion result we show that if, in addition, the norm of $X$ is strictly convex, then the assumptions $T(A) \subseteq A$ and $T(B) \subseteq B$ imply the existence of $x_{0} \in A$ and $y_{0} \in B$ such that $x_{0}$ and $y_{0}$ are fixed points of $T$ and $\left\|x_{0}-y_{0}\right\|=\operatorname{dist}(A, B)$.

2000 Mathematics Subject Classification: Primary 47H10.

Key words and phrases: proximal normal structure, relatively nonexpansive mappings, proximal points, fixed points. 
Strict convexity is essential for the second result. The significance of these two results lies in the fact that the "relative nonexpansive" assumption is much weaker than the assumption that $T$ is nonexpansive; in fact, it does not even imply continuity of $T$. Also, in contrast to the results of [5] where the contractive conditions on the mappings force $A$ and $B$ to intersect, the interesting case here is when $A \cap B=\emptyset$. In the event that $A \cap B \neq \emptyset$ then the restriction of $T$ to $A \cap B$ is nonexpansive, and our first result yields the fixed point theorem of Kirk [3] as a special case.

A Krasnosel'skiu type iteration method for approximating the fixed points of relatively nonexpansive mappings is also given, and in Section 3 some related Hilbert space results are discussed.

To describe our results we need some definitions and notation. We shall say that a pair $(A, B)$ of sets in a Banach space satisfies a property if each of the sets $A$ and $B$ has that property. Thus $(A, B)$ is said to be convex if both $A$ and $B$ are convex; $(A, B) \subseteq(C, D) \Leftrightarrow A \subseteq C$ and $B \subseteq D$, etc. We shall also adopt the notation

$$
\begin{aligned}
\delta(A, B) & =\sup \{\|x-y\|: x \in A, y \in B\} ; \\
\delta(x, A) & =\sup \{\|x-y\|: y \in A\} ; \\
\operatorname{dist}(A, B) & =\inf \{\|x-y\|: x \in A, y \in B\} .
\end{aligned}
$$

Definition 1.1. A pair $(A, B)$ of subsets of a normed linear space is said to be a proximal pair if for each $(x, y) \in A \times B$ there exists $\left(x^{\prime}, y^{\prime}\right) \in A \times B$ such that

$$
\left\|x-y^{\prime}\right\|=\left\|x^{\prime}-y\right\|=\operatorname{dist}(A, B) .
$$

Definition 1.2. A convex pair $\left(K_{1}, K_{2}\right)$ in a Banach space is said to have proximal normal structure if for any closed, bounded, convex proximal pair $\left(H_{1}, H_{2}\right) \subseteq\left(K_{1}, K_{2}\right)$ for which $\operatorname{dist}\left(H_{1}, H_{2}\right)=\operatorname{dist}\left(K_{1}, K_{2}\right)$ and $\delta\left(H_{1}, H_{2}\right)>\operatorname{dist}\left(H_{1}, H_{2}\right)$, there exists $\left(x_{1}, x_{2}\right) \in H_{1} \times H_{2}$ such that

$$
\delta\left(x_{1}, H_{2}\right)<\delta\left(H_{1}, H_{2}\right), \quad \delta\left(x_{2}, H_{1}\right)<\delta\left(H_{1}, H_{2}\right) .
$$

Notice that the pair $(K, K)$ has proximal normal structure if and only if $K$ has normal structure in the sense of Brodskii and Milman (cf. [3] and [1]). This can be seen by taking $K_{1}=K_{2}$ and $H_{1}=H_{2}$ in Definition 1.2, and observing that $\delta\left(H_{1}, H_{1}\right)=\operatorname{diam}\left(H_{1}\right)$ and $\operatorname{dist}\left(H_{1}, H_{1}\right)=0$. If $\delta\left(x_{1}, H_{1}\right)<$ $\delta\left(H_{1}, H_{1}\right)$ then $x_{1}$ is a nondiametral point of $H_{1}$.

2. Main results. We will show below that every convex pair in a uniformly convex Banach space has proximal normal structure, as do compact convex pairs in an arbitrary Banach space. First, however, we turn to our applications. 
TheOREM 2.1. Let $(A, B)$ be a nonempty, weakly compact convex pair in a Banach space, and suppose $(A, B)$ has proximal normal structure. Let $T: A \cup B \rightarrow A \cup B$ satisfy

(i) $T(A) \subseteq B$ and $T(B) \subseteq A$;

(ii) $\|T x-T y\| \leq\|x-y\|$ for $x \in A, y \in B$. Then there exists $(x, y) \in A \times B$ such that $\|x-T x\|=\|y-T y\|=\operatorname{dist}(A, B)$.

We also have the following fixed point result for relatively nonexpansive mappings. This requires the added assumption of strict convexity on the underlying space.

TheOREM 2.2. Let $(A, B)$ be a nonempty, weakly compact convex pair in a strictly convex Banach space, and suppose $(A, B)$ has proximal normal structure. Suppose $T: A \cup B \rightarrow A \cup B$ satisfies

(i) $T(A) \subseteq A$ and $T(B) \subseteq B$;

(ii) $\|T x-T y\| \leq\|x-y\|$ for $x \in A, y \in B$.

Then there exist $x_{0} \in A$ and $y_{0} \in B$ such that

$$
T x_{0}=x_{0}, \quad T y_{0}=y_{0}, \quad \text { and } \quad\left\|x_{0}-y_{0}\right\|=\operatorname{dist}(A, B) .
$$

Before proving the theorems, we introduce some more notation. Let $A$ and $B$ be subsets of a normed linear space $X$. The pair $(x, y) \in A \times B$ is said to be proximal in $(A, B)$ if $\|x-y\|=\operatorname{dist}(A, B)$. We use $\left(A_{0}, B_{0}\right)$ to denote the proximal pair obtained from $(A, B)$ upon setting

$$
\begin{aligned}
& A_{0}=\left\{x \in A:\left\|x-y^{\prime}\right\|=\operatorname{dist}(A, B) \text { for some } y^{\prime} \in B\right\}, \\
& B_{0}=\left\{y \in B:\left\|x^{\prime}-y\right\|=\operatorname{dist}(A, B) \text { for some } x^{\prime} \in A\right\} .
\end{aligned}
$$

In particular, if the pair $(A, B)$ is nonempty, weakly compact and convex, so also is the pair $\left(A_{0}, B_{0}\right)$, and moreover $\operatorname{dist}\left(A_{0}, B_{0}\right)=\operatorname{dist}(A, B)$. For details, see [4]. Also we use $B(x ; r)$ to denote the closed ball centered at $x \in X$ with radius $r \geq 0$.

Proof of Theorem 2.1. The theorem is trivial (via the theorem of [3]) if $A \cap B \neq \emptyset$, so we assume $\operatorname{dist}(A, B)>0$. Let $\left(A_{0}, B_{0}\right)$ be the proximal pair associated with $(A, B)$ as in (2.1). As we have just observed, $A_{0}$ and $B_{0}$ are weakly compact and convex, and $\operatorname{dist}\left(A_{0}, B_{0}\right)=\operatorname{dist}(A, B)$. Let $x \in A_{0}$. Then there exists $z \in B_{0}$ such that $\|x-z\|=\operatorname{dist}(A, B)$. Thus

$$
\|T x-T z\| \leq\|x-z\|=\operatorname{dist}(A, B) .
$$

This implies $T x \in B_{0}$; hence $T\left(A_{0}\right) \subseteq B_{0}$. Similarly, $T\left(B_{0}\right) \subseteq A_{0}$. Also

$$
\|T x-T y\| \leq\|x-y\| \quad \text { for } x \in A_{0}, y \in B_{0} .
$$

Clearly $\left(A_{0}, B_{0}\right)$ also has proximal normal structure. Now let $\Gamma$ denote the collection of all nonempty subsets $F$ of $A_{0} \cup B_{0}$ for which $F \cap A_{0}$ and $F \cap B_{0}$ 
are nonempty, closed and convex,

$$
T\left(F \cap A_{0}\right) \subseteq F \cap B_{0}, \quad T\left(F \cap B_{0}\right) \subseteq F \cap A_{0},
$$

and $\operatorname{dist}\left(F \cap A_{0}, F \cap B_{0}\right)=\operatorname{dist}(A, B)$. Since $A_{0} \cup B_{0} \in \Gamma, \Gamma$ is nonempty.

Let $\left\{F_{\alpha}\right\}_{\alpha \in J}$ be a descending chain in $\Gamma$, and let $F_{0}=\bigcap_{\alpha} F_{\alpha}$. Then $F_{0} \cap A_{0}=\bigcap_{\alpha}\left(F_{\alpha} \cap A_{0}\right)$, so $F_{0} \cap A_{0}$ is nonempty, closed and convex. Similarly $F_{0} \cap B_{0}$ is nonempty, closed and convex. Also

$$
T\left(F_{0} \cap A_{0}\right) \subseteq F_{0} \cap B_{0}, \quad T\left(F_{0} \cap B_{0}\right) \subseteq F_{0} \cap A_{0} .
$$

To show that $F_{0} \in \Gamma$ we only need to show that $\operatorname{dist}\left(F_{0} \cap A_{0}, F_{0} \cap B_{0}\right)=$ $\operatorname{dist}(A, B)$. However, for each $\alpha \in J$ it is possible to select $x_{\alpha} \in F_{\alpha} \cap A_{0}$ and $y_{\alpha} \in F_{\alpha} \cap B_{0}$ such that

$$
\left\|x_{\alpha}-y_{\alpha}\right\|=\operatorname{dist}(A, B) .
$$

It is also possible to choose weakly convergent subnets $\left\{x_{\alpha^{\prime}}\right\}$ and $\left\{y_{\alpha^{\prime}}\right\}$ (with the same indices), say weak- $\lim _{\alpha^{\prime}} x_{\alpha^{\prime}}=x$ and weak- $\lim _{\alpha^{\prime}} y_{\alpha^{\prime}}=y$. Then clearly $x \in F_{0} \cap A_{0}$ and $y \in F_{0} \cap B_{0}$. By weak lower semicontinuity of the norm,

$$
\|x-y\| \leq \operatorname{dist}(A, B)
$$

hence

$$
\operatorname{dist}(A, B) \leq \operatorname{dist}\left(F_{0} \cap A_{0}, F_{0} \cap B_{0}\right) \leq\|x-y\| \leq \operatorname{dist}(A, B) .
$$

Since every chain in $\Gamma$ is bounded below by a member of $\Gamma$, Zorn's lemma implies that $\Gamma$ has a minimal element, say $K$. Let $K_{1}=K \cap A_{0}$ and $K_{2}=K \cap B_{0}$. Observe that if

$$
\delta\left(K_{1}, K_{2}\right)=\operatorname{dist}\left(K_{1}, K_{2}\right),
$$

then $\|x-T x\|=\operatorname{dist}\left(K_{1}, K_{2}\right)=\operatorname{dist}(A, B)$ for any $x \in K_{1}$, and we are finished. So we may suppose that

$$
\delta\left(K_{1}, K_{2}\right)>\operatorname{dist}\left(K_{1}, K_{2}\right) .
$$

We complete the proof by showing that this leads to a contradiction.

Since $K$ is minimal it follows that $\left(K_{1}, K_{2}\right)$ is a proximal pair in $\left(A_{0}, B_{0}\right)$. By proximal normal structure there exist $\left(y_{1}, y_{2}\right) \in K_{1} \times K_{2}$ and $\beta \in(0,1)$ such that

$$
\delta\left(y_{1}, K_{2}\right) \leq \beta \delta\left(K_{1}, K_{2}\right), \quad \delta\left(y_{2}, K_{1}\right) \leq \beta \delta\left(K_{1}, K_{2}\right) .
$$

Since $\left(K_{1}, K_{2}\right)$ is a proximal pair there exists $\left(y_{1}^{\prime}, y_{2}^{\prime}\right) \in K_{1} \times K_{2}$ such that

$$
\left\|y_{1}-y_{2}^{\prime}\right\|=\left\|y_{2}-y_{1}^{\prime}\right\|=\operatorname{dist}\left(K_{1}, K_{2}\right) .
$$

So for any $z \in K_{2}$,

$$
\begin{aligned}
\left\|\frac{y_{1}+y_{1}^{\prime}}{2}-z\right\| & \leq\left\|\frac{y_{1}-z}{2}\right\|+\left\|\frac{y_{1}^{\prime}-z}{2}\right\| \\
& \leq \beta \delta\left(K_{1}, K_{2}\right) / 2+\delta\left(K_{1}, K_{2}\right) / 2=\alpha \delta\left(K_{1}, K_{2}\right),
\end{aligned}
$$


where $\alpha=(1+\beta) / 2 \in(0,1)$. Let $x_{1}=\left(y_{1}+y_{1}^{\prime}\right) / 2$ and similarly $x_{2}=$ $\left(y_{2}+y_{2}^{\prime}\right) / 2$. Then

$$
\delta\left(x_{1}, K_{2}\right) \leq \alpha \delta\left(K_{1}, K_{2}\right), \quad \delta\left(x_{2}, K_{1}\right) \leq \alpha \delta\left(K_{1}, K_{2}\right),
$$

and $\left\|x_{1}-x_{2}\right\|=\operatorname{dist}\left(K_{1}, K_{2}\right)$. Define

$$
\begin{aligned}
& L_{1}=\left\{x \in K_{1}: \delta\left(x, K_{2}\right) \leq \alpha \delta\left(K_{1}, K_{2}\right)\right\}, \\
& L_{2}=\left\{y \in K_{2}: \delta\left(y, K_{1}\right) \leq \alpha \delta\left(K_{1}, K_{2}\right)\right\} .
\end{aligned}
$$

Then $L_{i}$ is a nonempty closed convex subset of $K_{i}, i=1,2$, and since $x_{1} \in L_{1}$ and $x_{2} \in L_{2}, \operatorname{dist}\left(L_{1}, L_{2}\right)=\operatorname{dist}\left(K_{1}, K_{2}\right)(=\operatorname{dist}(A, B))$.

Now let $x \in L_{1}, z \in K_{2}$. Then $\|T x-T z\| \leq\|x-z\| \leq \alpha \delta\left(K_{1}, K_{2}\right)$. This implies

$$
T\left(K_{2}\right) \subseteq B\left(T x ; \alpha \delta\left(K_{1}, K_{2}\right)\right) \cap K_{1}:=K_{1}^{\prime} .
$$

Clearly $K_{1}^{\prime}$ is closed and convex. Also, if $y \in K_{2}$ satisfies $\|x-y\|=\operatorname{dist}(A, B)$ then $\|T x-T y\|=\operatorname{dist}\left(K_{1}, K_{2}\right)$. Since $T y \in K_{1}^{\prime}$, we conclude $\operatorname{dist}\left(K_{1}^{\prime}, K_{2}\right)=$ $\operatorname{dist}(A, B)$. Therefore $K_{1}^{\prime} \cup K_{2} \in \Gamma$, and by minimality of $K$ it must be the case that $K_{1}^{\prime}=K_{1}$; hence $K_{1} \subseteq B\left(T x ; \alpha \delta\left(K_{1}, K_{2}\right)\right)$ and since $x \in L_{1}$ was arbitrary this proves $T\left(L_{1}\right) \subseteq L_{2}$. Similarly $T\left(L_{2}\right) \subseteq L_{1}$. Therefore $L_{1} \cup L_{2} \in \Gamma$. But $\delta\left(L_{1}, L_{2}\right) \leq \alpha \delta\left(K_{1}, K_{2}\right)$, and this contradicts the minimality of $K$.

Proof of Theorem 2.2. Let $\left(A_{0}, B_{0}\right)$ be the proximal pair associated with $(A, B)$ and choose $x \in A_{0}$. Then there exists $z \in B_{0}$ such that $\|x-z\|=$ $\operatorname{dist}(A, B)$, and moreover $\|T x-T z\|=\operatorname{dist}(A, B)$. Thus $T: A_{0} \rightarrow A_{0}$. Similarly $T: B_{0} \rightarrow B_{0}$. Now let $\Gamma$ denote the collection of all nonempty subsets $F$ of $A_{0} \cup B_{0}$ for which $F \cap A_{0}$ and $F \cap B_{0}$ are nonempty, closed and convex,

$$
T\left(F \cap A_{0}\right) \subseteq F \cap A_{0}, \quad T\left(F \cap B_{0}\right) \subseteq F \cap B_{0},
$$

and $\operatorname{dist}\left(F \cap A_{0}, F \cap B_{0}\right)=\operatorname{dist}(A, B)$. Since $A_{0} \cup B_{0} \in \Gamma, \Gamma$ is nonempty. Proceed as in the proof of Theorem 2.1 to show that $\Gamma$ has a minimal element $K$. Let $K_{1}=K \cap A_{0}$ and $K_{2}=K \cap B_{0}$. First, suppose one of the sets is a singleton, say $K_{1}=\{x\}$. Then $T x=x$, and if $y$ is the unique point of $K_{2}$ for which $\|x-y\|=\operatorname{dist}\left(K_{1}, K_{2}\right)$ it must be the case that $T y=y$. Since $\|y-x\|=\operatorname{dist}(A, B)$, we are finished. So we may suppose both $K_{1}$ and $K_{2}$ have positive diameter, and because the space is strictly convex this in turn implies that

$$
\delta\left(K_{1}, K_{2}\right)>\operatorname{dist}\left(K_{1}, K_{2}\right) .
$$

We complete the proof by showing that this leads to a contradiction.

Since $\left(A_{0}, B_{0}\right)$ has proximal normal structure, we may define $L_{1}$ and $L_{2}$ as in the proof of Theorem 2.1. Choose $x \in L_{1}$. For any $z \in K_{2}$,

$$
\|T x-T z\| \leq\|x-z\| \leq \alpha \delta\left(K_{1}, K_{2}\right),
$$


and this implies

$$
T\left(K_{2}\right) \subseteq B\left(T x ; \alpha \delta\left(K_{1}, K_{2}\right)\right) \cap K_{2} .
$$

By minimality of $K$ it follows that $K_{2} \subseteq B\left(T x ; \alpha \delta\left(K_{1}, K_{2}\right)\right)$, and this in turn implies $\delta\left(T x, K_{2}\right) \leq \alpha \delta\left(K_{1}, K_{2}\right)$. Therefore $T\left(L_{1}\right) \subseteq L_{1}$. Similarly $T\left(L_{2}\right) \subseteq L_{2}$. Since $L_{1}$ and $L_{2}$ are, respectively, nonempty closed convex subsets of $K_{1}$ and $K_{2}$, and since $\delta\left(L_{1}, L_{2}\right) \leq \alpha \delta\left(K_{1}, K_{2}\right)$ for $\alpha<1$, this contradicts the minimality of $K$.

REMARK. The strict convexity assumption is essential in Theorem 2.2. To see this, it suffices to consider compact convex sets $A$ and $B$ in $\ell_{\infty}$ that have the property that $\|x-y\| \equiv d>0$ for $x \in A$ and $y \in B$. Then $(A, B)$ has proximal normal structure (vacuously). Any (even discontinuous) mapping $T: A \cup B \rightarrow A \cup B$ with $T(A) \subseteq A$ and $T(B) \subseteq B$ satisfies the assumptions of Theorem 2.2, but in general such a mapping need not have fixed points.

Now let $X$ be a uniformly convex Banach space with modulus of convexity $\delta$. Then $\delta(\varepsilon)>0$ for $\varepsilon>0$. Moreover, if $x, y, p \in X, R>0$, and $r \in[0,2 R]$, we have

$$
\left.\begin{array}{rl}
\|x-p\| & \leq R \\
\|y-p\| & \leq R \\
\|x-y\| & \geq r
\end{array}\right\} \Rightarrow\left\|\frac{x+y}{2}-p\right\| \leq\left(1-\delta\left(\frac{r}{R}\right)\right) R .
$$

It is well known that all uniformly convex Banach spaces have normal structure. They in fact have proximal normal structure.

Proposition 2.1. Every bounded closed convex pair in a uniformly convex Banach space $X$ has proximal normal structure.

Proof. Let $\left(H_{1}, H_{2}\right)$ be a bounded closed convex proximal pair in $X$, and suppose $\delta\left(H_{1}, H_{2}\right)>\operatorname{dist}\left(H_{1}, H_{2}\right)$. Choose $x, y \in H_{1}$ with $x \neq y$. Then if $\widetilde{x}, \widetilde{y} \in H_{2}$ satisfy

$$
\|x-\widetilde{x}\|=\|y-\widetilde{y}\|=\operatorname{dist}\left(H_{1}, H_{2}\right),
$$

it follows (by strict convexity) that $\widetilde{x} \neq \widetilde{y}$, and

$$
\left\|\frac{x+y}{2}-\frac{\widetilde{x}+\widetilde{y}}{2}\right\|=\operatorname{dist}\left(H_{1}, H_{2}\right) .
$$

Take $\varepsilon=\min \{\|x-y\|,\|\widetilde{x}-\widetilde{y}\|\}$. For any $z_{1} \in H_{2}$,

$$
\left\|x-z_{1}\right\| \leq \delta\left(H_{1}, H_{2}\right), \quad\left\|y-z_{1}\right\| \leq \delta\left(H_{1}, H_{2}\right) .
$$

Then if $\alpha=1-\delta\left(\varepsilon / \delta\left(H_{1}, H_{2}\right)\right)$ we have

$$
\left\|\frac{x+y}{2}-z_{1}\right\| \leq \alpha \delta\left(H_{1}, H_{2}\right) .
$$


Similarly if $z_{2} \in H_{1}$ then

$$
\left\|\frac{\widetilde{x}+\widetilde{y}}{2}-z_{2}\right\| \leq \alpha \delta\left(H_{1}, H_{2}\right)
$$

and the result follows.

Corollary 2.1. Let $(A, B)$ be a nonempty bounded closed convex pair in a uniformly convex Banach space. Let $T: A \cup B \rightarrow A \cup B$ satisfy

(i) $T(A) \subseteq B$ and $T(B) \subseteq A$;

(ii) $\|T x-T y\| \leq\|x-y\|$ for $x \in A, y \in B$.

Then there exists $(x, y) \in A \times B$ such that $\|x-T x\|=\|y-T y\|=\operatorname{dist}(A, B)$.

Corollary 2.2. Let $(A, B)$ be a nonempty bounded closed convex pair in a uniformly convex Banach space. Let $T: A \cup B \rightarrow A \cup B$ satisfy

(i) $)^{\prime} T(A) \subseteq A$ and $T(B) \subseteq B$;

(ii) $\|T x-T y\| \leq\|x-y\|$ for $x \in A, y \in B$.

Then there exist $x \in A$ and $y \in B$ such that $T x=x, T y=y$, and $\|x-y\|=$ $\operatorname{dist}(A, B)$.

Next we show that Krasnosel'skiı's iteration process (cf. [6]) yields a convergence result if $X$ is uniformly convex. In this theorem $A_{0}$ is the set in the proximal pair $\left(A_{0}, B_{0}\right)$ associated with the pair $(A, B)$ as described above.

TheOREM 2.3. Let $A$ and $B$ be nonempty bounded closed convex subsets of a uniformly convex Banach space and suppose $T: A \cup B \rightarrow A \cup B$ satisfies

(i) $T(A) \subseteq A$ and $T(B) \subseteq B$;

(ii) $\|T x-T y\| \leq\|x-y\|$ for $x \in A, y \in B$.

Let $x_{0} \in A_{0}$, and define $x_{n+1}=\left(x_{n}+T x_{n}\right) / 2, n=1,2, \ldots$ Then

$$
\lim _{n}\left\|x_{n}-T x_{n}\right\|=0 \text {. }
$$

Moreover, if $T(A)$ lies in a compact set, then $\left\{x_{n}\right\}$ converges to a fixed point of $T$.

Proof. If $\operatorname{dist}(A, B)=0$, then $A_{0}=B_{0}=A \cap B$ and the conclusion follows from a well known theorem of Ishikawa [2] and the fact that $T$ : $A \cap B \rightarrow A \cap B$ is nonexpansive. So we assume $\operatorname{dist}(A, B)>0$. By Theorem 2.2 there exists $y \in B_{0}$ such that $T y=y$. Since

$$
\begin{aligned}
\left\|x_{n+1}-y\right\| & =\left\|\frac{x_{n}+T x_{n}}{2}-\frac{y+T y}{2}\right\| \\
& \leq\left\|x_{n}-y\right\| / 2+\left\|T x_{n}-T y\right\| / 2 \leq\left\|x_{n}-y\right\|,
\end{aligned}
$$

$\left\{\left\|x_{n}-y\right\|\right\}$ is nonincreasing and $\lim _{n}\left\|x_{n}-y\right\|=d>0$. Suppose there exists a subsequence $\left\{x_{n_{k}}\right\}$ of $\left\{x_{n}\right\}$ and an $\varepsilon>0$ such that $\left\|x_{n_{k}}-T x_{n_{k}}\right\| \geq \varepsilon>0$ for 
all $k$. Since the modulus of convexity $\delta$ of $X$ is an increasing (and continuous) function it is possible to choose $\xi>0$ so small that

$$
\left(1-\delta\left(\frac{\varepsilon}{d+\xi}\right)\right)(d+\xi)<d
$$

Then if $k$ is chosen so that $\left\|x_{n_{k}}-y\right\| \leq d+\xi$, we have the contradiction:

$$
\left\|y-x_{n_{k}+1}\right\|=\left\|y-\frac{x_{n_{k}}+T x_{n_{k}}}{2}\right\| \leq\left(1-\delta\left(\frac{\varepsilon}{d+\xi}\right)\right)(d+\xi) .
$$

This proves that $\lim _{n}\left\|x_{n}-T x_{n}\right\|=\lim _{n}\left\|x_{n}-x_{n+1}\right\|=0$. If $T(A)$ is compact then $\left\{x_{n}\right\}$ has a subsequence $\left\{x_{n_{k}}\right\}$ that converges to a point $z \in A$. Also $\left\{x_{n_{k}+1}\right\}$ and $\left\{T x_{n_{k}}\right\}$ converge to $z$. Let $D=\operatorname{dist}(A, B)$ and choose $w \in B_{0}$ so that $\|z-w\|=D$. We now have $\left\|x_{n_{k}}-w\right\| \rightarrow\|z-w\|=D$, and by (ii),

$$
\left\|x_{n_{k}}-w\right\| \geq\left\|x_{n_{k}+1}-T w\right\| \rightarrow\|z-T w\|
$$

so $\|z-T w\|=D$. By strict convexity of the norm, $T w=w$, and by (ii), $T z=z$ because $z$ is the unique point of $A$ which is nearest to $w$.

It is possible to give simple examples (even on the real line) to show that the assumption $x_{0} \in A_{0}$ is necessary in Theorem 2.3.

Finally, we have the following result, which illustrates that proximal normal structure is similar to normal structure in another way.

Proposition 2.2. Every compact convex pair $\left(K_{1}, K_{2}\right)$ in a Banach space has proximal normal structure.

Proof. Let $\left(H_{1}, H_{2}\right)$ be any bounded closed convex proximal pair contained in $\left(K_{1}, K_{2}\right)$ for which $\delta\left(H_{1}, H_{2}\right)>\operatorname{dist}\left(H_{1}, H_{2}\right)$, and suppose $\delta\left(x, H_{2}\right)$ $=\delta\left(H_{1}, H_{2}\right)$ for each $x \in H_{1}$. Let $x_{0} \in H_{1}$. Then there exists $y_{0} \in H_{2}$ such that $\left\|x_{0}-y_{0}\right\|=\delta\left(H_{1}, H_{2}\right)$. Since $\left(H_{1}, H_{2}\right)$ is a proximal pair there exists $x_{1} \in H_{1}$ such that $\left\|x_{1}-y_{0}\right\|=\operatorname{dist}\left(H_{1}, H_{2}\right)$. Therefore

$$
\left\|x_{1}-x_{0}\right\| \geq\left\|x_{0}-y_{0}\right\|-\left\|x_{1}-y_{0}\right\|=\delta\left(H_{1}, H_{2}\right)-\operatorname{dist}\left(H_{1}, H_{2}\right) .
$$

Choose $y_{1} \in H_{2}$ so that $\left\|\left(x_{1}+x_{0}\right) / 2-y_{1}\right\|=\delta\left(H_{1}, H_{2}\right)$. This implies

$$
\left\|x_{1}-y_{1}\right\|=\left\|x_{0}-y_{1}\right\|=\delta\left(H_{1}, H_{2}\right) .
$$

Having chosen $\left\{x_{1}, \ldots, x_{n}\right\}$ in $H_{1}$, take $y_{n} \in H_{2}$ so that

$$
\left\|\frac{x_{1}+\cdots+x_{n}}{n}-y_{n}\right\|=\delta\left(H_{1}, H_{2}\right) \text {. }
$$

Now choose $x_{n+1} \in H_{1}$ so that $\left\|x_{n+1}-y_{n}\right\|=\operatorname{dist}\left(H_{1}, H_{2}\right)$. Having defined the sequence $\left\{x_{n}\right\}$, observe that since $\left\|x_{i}-y_{n}\right\|=\delta\left(H_{1}, H_{2}\right)$ for all $i=$ $1, \ldots, n$, we have

$$
\left\|x_{n+1}-x_{i}\right\| \geq\left\|x_{i}-y_{n}\right\|-\left\|x_{n+1}-y_{n}\right\|=\delta\left(H_{1}, H_{2}\right)-\operatorname{dist}\left(H_{1}, H_{2}\right)
$$


for $i=1, \ldots, n$. Since $\delta\left(H_{1}, H_{2}\right)-\operatorname{dist}\left(H_{1}, H_{2}\right)>0$ this contradicts the compactness of $H_{1}$. Therefore there exists $x \in H_{1}$ such that $\delta\left(x, H_{2}\right)<$ $\delta\left(H_{1}, H_{2}\right)$. Similarly there exists $y \in H_{2}$ such that $\delta\left(y, H_{1}\right)<\delta\left(H_{1}, H_{2}\right)$.

3. Hilbert spaces. We now examine the results of the previous section in a Hilbert space setting. Suppose $A$ is a nonempty closed convex subset of a real Hilbert space $X$. For any $x \in X$ let $P_{A} x$ denote the unique point of $A$ for which

$$
\left\|x-P_{A} x\right\|=\operatorname{dist}(x, A) .
$$

It is well known that $P_{A}$ is nonexpansive and characterized by the inequality

$$
\left\langle z-P_{A} x, P_{A} x-x\right\rangle \geq 0 \quad \text { for all } x \in X \text { and } z \in A .
$$

The next observation provides an example of a relatively nonexpansive mapping.

Proposition 3.1. Let $A$ and $B$ be two closed and convex subsets of a Hilbert space $X$, and define $P: A \cup B \rightarrow A \cup B$ to be the restriction of $P_{B}$ on $A$ and the restriction of $P_{A}$ on $B$. Then $P(A) \subseteq B, P(B) \subseteq A$, and $\|P x-P y\| \leq\|x-y\|$ for $x \in A$ and $y \in B$.

Proof. Suppose $x \in A$ and $y \in B$. Then by (3.1),

$$
\left\langle y-P_{B} x, P_{B} x-x\right\rangle \geq 0, \quad\left\langle x-P_{A} y, P_{A} y-y\right\rangle \geq 0 .
$$

Adding the above two terms, we have

$$
\left\langle y-P_{B} x, P_{B} x-x\right\rangle-\left\langle x-P_{A} y, y-P_{A} y\right\rangle \geq 0 .
$$

Simple calculations yield

$$
\begin{aligned}
& \left\langle y-P_{B} x, P_{B} x+P_{A} y-(x+y)\right\rangle+\left\langle y-x+P_{A} y-P_{B} x, y-P_{A} y\right\rangle \geq 0, \\
& \left\langle y-x+P_{A} y-P_{B} x, P_{B} x-x\right\rangle+\left\langle x-P_{A} y, P_{B} x+P_{A} y-(x+y)\right\rangle \geq 0 .
\end{aligned}
$$

Adding again we have

$$
\begin{aligned}
& \left\langle\left(P_{A} y+P_{B} x\right)-(x+y),(x+y)-\left(P_{B} x+P_{A} y\right)\right\rangle \\
& +\left\langle y-x+P_{A} y-P_{B} x, y-x+P_{B} x-P_{A} y\right\rangle \geq 0 .
\end{aligned}
$$

Thus

$$
\left\|P_{B} x-P_{A} y\right\|^{2} \leq\|x-y\|^{2}-\left\|(x+y)-\left(P_{A} y+P_{B} x\right)\right\|^{2},
$$

which implies $\left\|P_{B} x-P_{A} y\right\| \leq\|x-y\|$.

Now suppose $T: A \cup B \rightarrow A \cup B$ ( $A$ and $B$ as above) satisfies $T(A) \subseteq A$ and $T(B) \subseteq B$, and suppose

$$
\|T x-T y\| \leq\|x-y\| \quad \text { for } x \in A \text { and } y \in B .
$$

Define $U: A \cup B \rightarrow A \cup B$ by setting

$$
U x=P_{B} T x \quad \text { if } x \in A \quad \text { and } \quad U y=P_{A} T y \quad \text { if } y \in B .
$$


Then by Theorem 2.1 there exists $x_{0} \in A_{0}$ such that $\left\|x_{0}-P_{B} T x_{0}\right\|=$ $\operatorname{dist}(A, B)$. Since $\left\|x_{0}-P_{B} x_{0}\right\|=\operatorname{dist}(A, B),(3.3)$ implies $\left\|T x_{0}-T P_{B} x_{0}\right\|$ $=\operatorname{dist}(A, B)$. But this in turn implies $\left\|T x_{0}-P_{B} T x_{0}\right\|=\operatorname{dist}(A, B)$. This means that both $x_{0}$ and $T x_{0}$ are proximal points for $P_{B} T x_{0}$, so by uniqueness of proximal points, $T x_{0}=x_{0}$.

Thus in a Hilbert space setting Theorem 2.2 follows directly from Theorem 2.1. This does not appear to be true in general.

Similarly there is a more direct approach to Theorem 2.2 in a Hilbert space setting. The mapping $T$ of Theorem 2.2 need not be continuous on $A \cup B$, although it is fairly easy to see that it is continuous if $(A, B)$ is a proximal pair. In a Hilbert space setting $T$ is in fact nonexpansive on $A \cup B$ if $(A, B)$ is a proximal pair.

Proposition 3.2. Suppose $A$ and $B$ are bounded closed convex subsets of a Hilbert space, and suppose $A=A_{0}$ and $B=B_{0}$. Suppose $T: A \cup B \rightarrow$ $A \cup B$ satisfies

(i) $T(A) \subseteq A$ and $T(B) \subseteq B$;

(ii) $\|T x-T y\| \leq\|x-y\|$ for $x \in A, y \in B$.

Then $T$ is nonexpansive on $A \cup B$.

Proof. Let $u, v \in A$ and let $d=\operatorname{dist}(A, B)$. Since $\left\|T P_{B} u-T u\right\| \leq$ $\left\|P_{B} u-u\right\|$ it must be the case that

$$
T\left(P_{B}(u)\right)=P_{B}(T(u)) .
$$

Also it is easy to see that the segment $\left[u, P_{B} u\right]$ is orthogonal to $[u, v]$. (This follows from the fact that $u$ is the point on the line passing through $u$ and $v$ which is nearest to $P_{B} u$.) Similarly the segment $\left[T u, P_{B} T u\right]$ is orthogonal to $[T u, T v]$. By the Pythagorean Theorem we have

$$
d^{2}+\|T u-T v\|^{2}=\left\|T P_{B} u-T v\right\|^{2} \leq\left\|P_{B} u-v\right\|^{2}=d^{2}+\|u-v\|^{2},
$$

from which $\|T u-T v\| \leq\|u-v\|$.

Proposition 3.2 in conjunction with the fixed point theorem for nonexpansive mappings immediately ensures the existence of a fixed point $x_{0}$ of $T$ in $A$, and the unique point $y_{0} \in B$ which is nearest to $x_{0}$ satisfies $T y_{0}=y_{0}$ and $\left\|x_{0}-y_{0}\right\|=\operatorname{dist}(A, B)$.

\section{References}

[1] M. S. Brodskiur and D. P. Milman, On the center of a convex set, Dokl. Akad. Nauk SSSR (N.S.) 59 (1948), 837-840 (in Russian).

[2] S. Ishikawa, Fixed points and iteration of a nonexpansive mapping in a Banach space, Proc. Amer. Math. Soc. 59 (1976), 65-71.

[3] W. A. Kirk, A fixed point theorem for mappings which do not increase distances, Amer. Math. Monthly 72 (1965), 1004-1006. 
[4] W. A. Kirk, S. Reich, and P. Veeramani, Proximinal retracts and best proximity pair theorems, Numer. Funct. Anal. Optim. 24 (2003), 851-862.

[5] W. A. Kirk, P. S. Srinivasan, and P. Veeramani, Fixed points for mappings satisfying cyclical contractive conditions, Fixed Point Theory 4 (2003), 79-89.

[6] M. A. Krasnosel'skiǔ, Two remarks on the method of successive approximations, Uspekhi Mat. Nauk (N.S.) 10 (1955), 123-127 (in Russian).

Department of Mathematics

Indian Institute of Technology, Madras

Chennai, India

E-mail: pvmani@iitm.ac.in
Department of Mathematics The University of Iowa Iowa City, IA 52242-1419, U.S.A. E-mail: kirk@math.uiowa.edu 